\title{
A narrative review of emergency use authorization versus full FDA approval and its effect on COVID-19 vaccination hesitancy
}

\author{
Thoyaja Koritala1 , Akbar Hussain ${ }^{2}$, Yelena Pleshkova ${ }^{3}$, Lavanya Dondapati4, \\ Raghavendra Tirupathi' ${ }^{5}$, Ali A Rabaan ${ }^{6,7}$, Abbas Al Mutair8,9,10, Saad Alhumaid ${ }^{11}$, \\ Jaffar A Al-Tawfiq ${ }^{12,13,14}$, Rahul Kashyap ${ }^{15}$ \\ ${ }^{1}$ Department of Internal Medicine, Mayo Clinic Health System, Mankato, MN, USA; \\ 2Department of Internal Medicine, Keystone Health, Chambersburg, PA, USA; \\ ${ }^{3}$ Department of Clinical Sciences, University of Houston College of Medicine, Houston, TX, USA; \\ ${ }^{4}$ Department of Internal Medicine, Baylor St. Luke's Medical Center, Houston, TX, USA; \\ ${ }^{5}$ Department of Medicine, Keystone Health, Penn state College of Medicine, Chambersburg, PA, USA; \\ ${ }^{6}$ Molecular Diagnostic Laboratory, Johns Hopkins Aramco Healthcare, Dhahran, Saudi Arabia; \\ ${ }^{7}$ Department of Public Health and Nutrition, University of Haripur, Haripur, Pakistan; \\ ${ }^{8}$ Research center, Almoosa Specialist Hospital, Al-Ahsa, Saudi Arabia; \\ ${ }^{9}$ College of Nursing, Princess Norah Bint Abdulrahman University, Riyadh, Saudi Arabia; \\ ${ }^{10}$ School of Nursing, Wollongong University, Wollongong NSW, Australia; \\ ${ }^{11}$ Administration of Pharmaceutical Care, Al-Ahsa Health Cluster, Ministry of Health, Al-Ahsa, Saudi Arabia; \\ ${ }^{12}$ Infectious Disease Unit, Specialty Internal Medicine and Infection Control Unit, Quality and Patient Safety, \\ Johns Hopkins Aramco Healthcare, Dhahran, Saudi Arabia; \\ ${ }^{13}$ Infectious Disease Division, Department of Medicine, Indiana University School of Medicine, Indianapolis, IN, USA; \\ ${ }^{14}$ Infectious Disease Division, Department of Medicine, Johns Hopkins University School of Medicine, \\ Baltimore, MD, USA; \\ ${ }^{15}$ Department of Anesthesiology and Peri-operative Medicine, Mayo Clinic, Rochester, MN, USA.
}

Article received 21 July, 2021; accepted 4 August, 2021

\section{SUMMARY}

COVID-19 pandemic affected the lives of many with its devastating mortality and morbidity. Acquisition of herd immunity is one way to mitigate the spread of infection. Many factors influence the acceptance of vaccination including the regulatory process of the vaccines. This review article will briefly summarize the Emergency Use Authorization, Full FDA Approval process and highlight how the key factors affecting the vaccination hesitancy, are being influenced by the lack of Full FDA Approval.

Keywords: COVID-19, emergency use authorization, full FDA approval, herd immunity, vaccine resistance.

\section{INTRODUCTION}

OVID-19, a novel Coronavirus, originated in China at the end of 2019. It has rapidly spread worldwide with crippling effects and disruptions across all facets of life resulting in high morbidity

Corresponding author

Thoyaja Koritala

E-mail: koritala.thoyaja@mayo.edu and mortality [1, 2]. As of June 2021, the number of deaths in the USA is approximating $>600,000$ and the struggle to control its resurgence continues (WHO 2021) [3]. The USA initiated Operation warp speed in April 2020 with a goal to rapidly develop vaccines to counter the spread of the virus and boost population immunity against the disease. The research finally led to the creation of vaccines in USA by Pfizer-BioNtech, Moderna and Johnson \& Johnson. These three companies 
applied for Emergency use authorization of their vaccines and the FDA granted approval. Emergency use authorization (EUA) is the process by which the FDA grants approval for use of medical products in times of public health emergencies like COVID-19 [4]. Vaccinations have been administered in a tiered way to give priority to vulnerable populations during phase 1 before subsequent equitable distribution to the public. Graham et al. indicated that vaccinations are the most effective way to develop herd immunity and that it needs $60-70 \%$ of the population to be vaccinated [5]. As of June 2021, $>50 \%$ of the USA population has been partially vaccinated and $>45 \%$ are fully vaccinated [6,7]. The inability to reach the desired level of herd immunity may cause the virus to mutate and enhance its virulence and transmissibility. The effects in Asian countries like India (9.9\% partially vaccinated and $2.6 \%$ fully vaccinated) have been devastating and mortality rates have increased in a matter of a few weeks [8].

We aim to highlight the contributory potential role of Emergency Use Authorization, rather than Full Authorization by FDA, in unwillingness of people to take COVID-19 vaccine in USA.

\section{Emergency use authorization}

Emergency use authorization was first introduced in 1938 under section 564 of the Federal Food Drug and Cosmetic act and was subsequently amended by the project Bioshield Act of 2004 [9, 10]. The passing of this act put forth the passage of the Pandemic and All-Hazards preparedness reauthorization act of 2013 [11]. FDA can grant approval for use of medical products and devices under the Emergency use Authorization.

\section{FDA Regulatory Approval Process for Vaccines}

Food and Drug Administration is a federal agency that oversees the safety, efficacy, and security of food safety, pharmaceutical drugs for humans and animals, vaccines, biological products, overthe-counter drugs, and nutritional supplements.
Vaccines are thoroughly evaluated by the FDA's Center for Biologics Evaluation and Research [12]. Every potential vaccine candidate is put through a thorough and exhaustive regulatory process before they are available for administration in the USA. The following are brief steps involved in the production of a new vaccine (Figure 1).

1) Research and Development: This is the first phase where potential vaccine candidates are screened for their efficacy and sometimes for their safety profiles as well.

2) Preclinical phase: This is the second phase where vaccine candidates developed in the lab are tested heavily on animals to see which among them are safe and effective for further testing in humans.

3) Clinical Phase: This is the phase when the best vaccine candidate will be tested in humans. Prior to administration of vaccine to human volunteers, the facility needs to apply for an Investigational New Drug application. FDA also needs to visit the facility and deem it to be a good manufacturing site for the scale up of the vaccine production. The Clinical Phase encompasses three phases which are each crucial to ascertain the vaccine efficacy, safety and the side effects attributed to the vaccine.

- Phase 1 (safety): This phase includes a small sample size of healthy volunteers which might range anywhere from 20-100. The whole purpose of this phase is to find the safety of the vaccine and a possible look toward any untoward side effects with different doses that are being administered. By the end of this phase an optimal dosage for the vaccine, its side effects and preliminary data of its efficacy can be seen.

- Phase 2 (immune response and safety): This phase is often characterized by randomized controlled studies on larger diverse patient populations with varying health predispositions. This phase investigates the efficacy of the vaccine as a whole by measuring the immune response elicited by the vaccine. Further along we can also in-

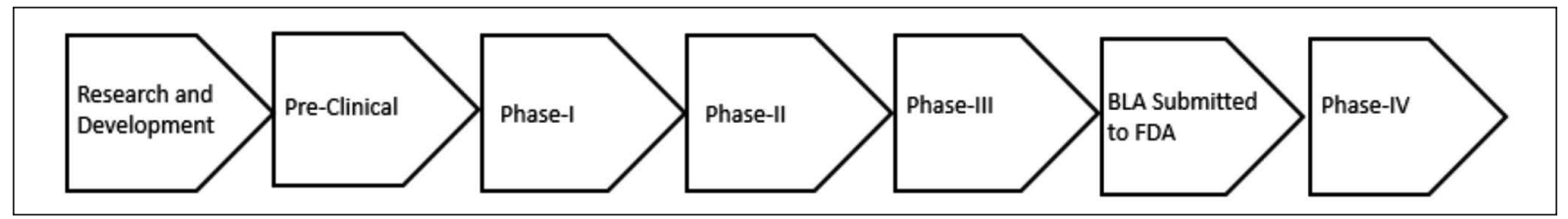

Figure 1 - Flow Chart Explaining Approval Process for Vaccination. 
vestigate the vaccine's safety and side effects in different patient populations with pre-existing conditions as well.

- Phase 3 (safety and efficacy): This is the final phase where the vaccine is administered in a large population to measure its safety and efficacy. Phase 3 studies enable effective comparison between vaccinated and control groups.

\section{Approval of Vaccine}

After Phase 3 studies, the manufacturers usually apply for a Biologics License Application (BLA). FDA approval for a BLA gives the manufacturer the license to market the vaccine in the USA. In short, the approval means that the vaccine has shown its efficacy with a good safety profile and is being manufactured under conditions which can assure the quality and consistency for the product.

- Phase 4: Once the vaccine is labelled with information regarding its use and potential side effects, the FDA keeps monitoring for additional information regarding the vaccine's risks, benefits and best use.

Emergency Use Authorization or EUA does make the vaccine pass through the same rigorous evaluations like other vaccines, including passing Phase 3 clinical trials, before it is authorized for the emergency use in populations [4].

\section{Willingness to take COVID-19 Vaccine with}

\section{the Emergency Use Authorization from FDA}

There has been a lot of skepticism around the acceptance of the COVID-19 vaccine and vaccination. This skepticism has been present and persisted since the inception of the vaccine, due to an accelerated development and production schedule. There have been several factors that have affected the willingness of segments of the US population to take the vaccine and are detailed below (Figure 2).

\section{Safety and Efficacy}

Although major doubts surrounding safety have been debunked, the emergence of life-threatening blood clots following vaccination with the Johnson \& Johnson vaccine in the USA has again brought the focus regarding the safety of the vaccines to the forefront [13, 14]. The Theory of Planned Behavior states that the emergence of potential side effects creates negative feedback which leads to

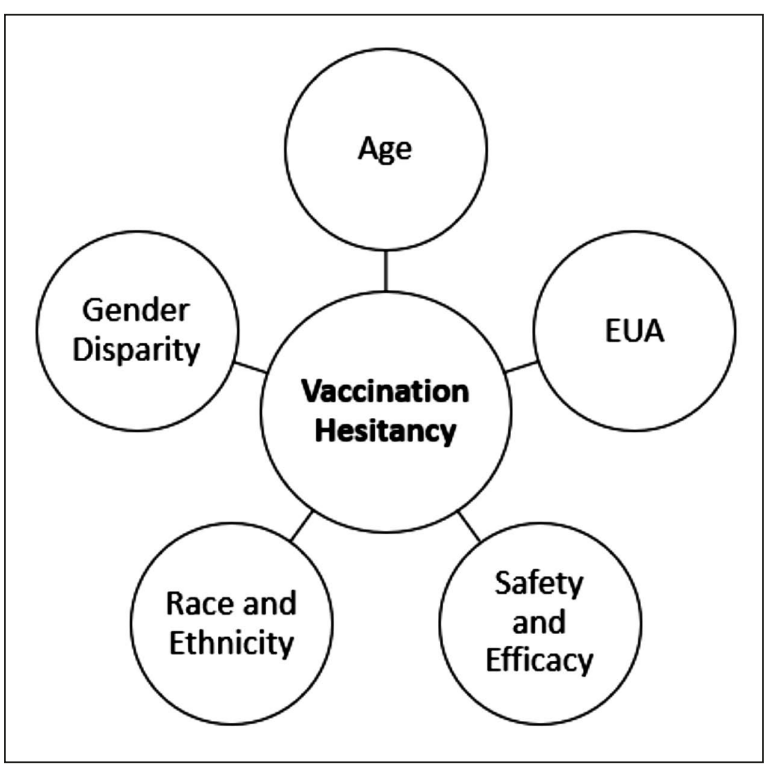

Figure 2 - Key Factors Influencing Vaccination Hesitancy.

resistance toward vaccinations in the public [15]. Historically, there has been resistance toward the use of non-approved drugs [16]. A full FDA approval on the other hand gives a detailed explanation of possible risks and benefits, as well as the proper use of the vaccine for the public. Once full FDA approval is granted to a vaccine, patients can be better informed about its risks and benefits.

\section{Gender disparity in acceptance toward vaccines with EUA}

Historically females are more resistant to being vaccinated, because of their belief in the efficacy, and risks associated with vaccines [17, 18]. However, data regarding COVID-19 vaccination demonstrates that females $(52.54 \%$ with one dose and $45.33 \%$ fully vaccinated) outnumber the males $(48.28 \%$ with one dose and $41.51 \%$ fully vaccinated) [19]. These results might be skewed toward females, as there were a greater number of females over 65 than males. However, the gap remains significant even when considering the higher life expectancy in females [20]. Females are likely to be more receptive of COVID-19 vaccinations because of their role as primary caregivers at home and recent research showing women with polycystic ovarian syndrome to be more severely affected by COVID-19 infections [21]. The reason why males are showing unwillingness to 
take vaccination might be due to the political divide and rhetoric behind the vaccines [22, 23]. We opine that EUA might be misconstrued as a governmental thrust on vaccinations and full approval might tone down that narrative and encourage people to take these vaccinations.

\section{Influence of race and ethnicity on resistance toward vaccination}

Race and ethnicity have had a marked effect on general vaccine acceptance. Both Black (29\%) and Hispanic (32\%) populations have had a lower vaccination rate compared to Whites $(43 \%)$ and Asians (54\%) [24]. This can be attributed to mistrust in the government and its policies [25]. Historical precedents such as the Tuskegee Syphilis study, further cements African American distrust in the government [26]. Based on the historic racial disparities, the use of EUA for vaccinations drives the mistrust deeper in these African American communities [27]. Institutional racism has also played a major role in the breakdown of trust due to the presence of healthcare inequity at several levels [28]. Communication plays a key role in trying to reduce vaccine resistance by promoting vaccination discussions through patients Primary Care providers. However, EUA in some ways pushes vaccinations away from these primary care providers to mass vaccination sites where an unfamiliar healthcare provider has this conversation. Full FDA approval, as opposed to EUA, might make hesitant groups more likely to accept vaccination at sites other than a primary care setting.

Hispanic populations are slightly better vaccinated than African American populations, but they still are lagging when compared to other populations [24]. A significant segment of the Hispanic populations are migrants who fear government actions regarding immigration violations and enforcement and thus avoid contact with governmental agencies [29]. EUA and the federal government's outreach in collecting information about vaccination might make these populations more hesitant to accept vaccination. Full FDA approval might alleviate the need for additional data collection and encourage vulnerable Hispanic populations to be vaccinated at a higher rate.

\section{Age groups and EUA}

It can be seen from the data available that the age group from 18-29 have the lowest COVID-19 vaccination rate $(48 \%)$ compared to 30 years and above [30]. This group tends to be a significant spreader of COVID-19 infection and their vaccination would help reduce the transmission of COVID-19 to vulnerable populations in the community [31]. A considerable proportion of this younger population $(14 \%)$ is willing to take the vaccination, if it is required [30]. It is likely that a significant number in this population is enrolled in higher education and most educational institutions, especially public institutions cannot be mandated to require vaccination without full FDA approval [32]. According to FDA rules EUA products cannot be mandated [33]. Full FDA approval can allow Educational Institutions to mandate COVID vaccinations similar to other vaccinations like measles and mumps etc. This is particularly important as it can trickle down further to school-going age groups, once approved. It has been seen that the willingness of parents to get their children vaccinated with EUA vaccines is around 51\% [34]. Although children are thought to be less likely than adults to develop severe COVID-19 symptoms, it has been seen that $33 \%$ of children aged $12-17$ have been developing severe symptoms often requiring intensive care unit hospitalizations [35, 36]. Although Pfizer and Moderna were granted EUA's for children 12-17 years old in May 2021, there has been a significant lag in vaccinations in this age group. The full approval of the vaccine by the FDA can encourage more children to be vaccinated, protecting them and other vulnerable populations interacting with the children.

\section{Application for Full FDA approval}

Pfizer and Moderna have applied for a Biological License Application in May and June of this year $[37,38]$. Once the Application is in place, it can take the FDA almost 10 months to grant full approval. Once a BLA for a vaccine is approved it can be sent directly to sites for administration [39]. Long timelines for approval can be risky as they promote vaccine hesitancy and can enable interim viral mutations affecting subsequent efficacy.

\section{CONCLUSIONS}

It has been seen that administered vaccinations have significantly reduced the number of COVID-19 infections. It can thus be inferred that COVID-19 vaccinations are highly effective [40]. Except 
for the Johnson \& Johnson vaccine, Pfizer-BioNtech and Moderna vaccines haven't had any significant life-threatening side effects reinforcing the safety of the authorized vaccines. However, the residual reluctance to be vaccinated is well noted. It would be encouraging to see an efficient Full approval for COVID-19 vaccination by the FDA, to encourage reluctant populations to be immunized and lead us toward the threshold of herd immunity required to overcome this Pandemic.

\section{Conflict of interest}

None of the co-authors have declared any conflict of interest. No funding is involved.

\section{Funding}

None.

\section{REFERENCES}

[1] Shah A, Kashyap R, Tosh P, Sampathkumar P, O'Horo JC. Guide to understanding the 2019 novel coronavirus. Mayo Clin Proc. 2020; 95 (4), 646-52.

[2] Domecq JP, Lal A, Sheldrick CR, et al. Outcomes of patients with coronavirus disease 2019 receiving organ support therapies: The International Viral Infection and Respiratory Illness Universal Study Registry. Crit Care Med. 2021; 49 (3), 437-48. Erratum in: Crit Care Med. 2021; 49 (5): e562.

[3] World Health Organization. WHO coronavirus (COVID-19) dashboard: Situation by region, country, territory \& area. Available at: https: / / covid19.who.int/ table?tableChartType=heat. [accessed 17/07/2021].

[4] Emergency Use Authorization for Vaccines Explained | FDA. Available at: https://www.fda.gov/ vaccines-blood-biologics/vaccines/emergency-use-authorization-vaccines-explained. [accessed 15/06/2021].

[5] Graham BS. Rapid COVID-19 vaccine development. Science. 2020; 368 (6494), 945-6.

[6] Our World in Data. Statistics and research: coronavirus (COVID-19) vaccinations. Available at: https:/ / ourworldindata.org/covid-vaccinations. [accessed 18/ 06/2021].

[7] Holder J. Tracking Coronavirus Vaccinations Around the World. Available at: https://www.nytimes.com/ interactive/2021/world / covid-vaccinations-tracker. html. [accessed 18/06/2021].

[8] Lovelace B Jr. WHO classifies triple-mutant Covid variant from India as global health risk. Available at: https: / / www.cnbc.com/2021/05/10/who-classifiestriple-mutant-covid-variant-from-india-as-globalhealth-risk-.html. [accessed 10/05/2021].

[9] Courtney B. FDA Emergency Use Authorizations (EUAs): what are they and what do they mean for the
H1N1 swine flu response? Biosecur Bioterror. 2009; 7 (2), 211-3.

[10] Nightingale SL, Prasher JM, Simonson S. Emergency Use Authorization (EUA) to enable use of needed products in civilian and military emergencies, United States. Emerg Infect Dis. 2007; 13 (7), 1046-51.

[11] Association of State and Territorial Health Officials. Emergency Use Authorization toolkit: Section 564 of the Federal Food, Drug, and Cosmetic Act. Available at: https://www.astho.org/Programs/Preparedness / Public-Health-Emergency-Law / Emergency-Use-Authorization-Toolkit/Section-564-of-the-Federal-Food,Drug,-and-Cosmetic-Act-Fact-Sheet/\#: :text=Overview,during $\% 20$ certain $\% 20$ types $\% 20$ of $\% 20$ emergencies. [accessed 18/06/2021].

[12] U.S. Food and Drug Administration. Vaccine development - 101. Available at: https://www.fda. gov/vaccines-blood-biologics / development-approval-process-cber/vaccine-development-101. [accessed 18/06/2021].

[13] Weintraub K. CDC reports 13 additional cases of blood clots linked to J\&J COVID-19 vaccine. All happened before 11-day pause in its use. Available at: https: / / www.usatoday.com/story / news / health / 2021/05/12/j-j-covid-19-vaccine-cdc-additional-casesblood-clots/5063485001/. [accessed 12/05/2021].

[14] Centers for Disease Control and Prevention. CDC recommends use of Johnson \& Johnson's Janssen COVID-19 vaccine resume. Available at: https://www.cdc. gov / coronavirus / 2019-ncov/vaccines / safety/JJUpdate.html. [accessed 13/07/2021].

[15] Ajzen I. The theory of planned behavior. Organizat Behav Hum Decis Proc. 1991; 50, 179-211.

[16] Paek HJ, Hilyard K, Freimuth VS, Barge JK, Mindlin M. Public support for government actions during a flu pandemic: lessons learned from a statewide survey. Health Promot Pract. 2008; 9 (4 Suppl), 60S-72S.

[17] Everett WW, Coffin SE, Zaoutis T, Halpern SD, Strom BL. Smallpox vaccination: a national survey of emergency health care providers. Acad Emerg Med. 2003; 10 (6), 606-11.

[18] Quinn SC, Kumar S, Freimuth VS, Kidwell K, Musa D. Public willingness to take a vaccine or drug under emergency use authorization during the 2009 H1N1 pandemic. Biosecur Bioterror. 2009; 7, 275-90.

[19] USA Facts. US coronavirus vaccine tracker. Available at: https: / / usafacts.org/visualizations / covid-vaccine-tracker-states / ?utm_source=google\&utm_medium =cpc\&utm_campaign=ND-COVID-Vaccine\&gclid=CjwKCAjwqcKFBhAhEiw AfEr7zZUIR2ipyEYPrhWwlzKOLUCJxdHoIp6ugE9ZAgmBCpEJBaE2JrYqcBoCD1gQAvD_BwE. [accessed 03/07/2021].

[20] Arias E, Tejada-Vera B, Ahmad F. Provisional life expectancy estimates for January through June, 2020. Available at: https://www.cdc.gov/nchs/data/vsrr/ VSRR10-508.pdf. [accessed 03/07/2021]. 
[21] Kyrou I, Karteris E, Robbins T, Chatha K, Drenos F, Randeva HS. Polycystic ovary syndrome (PCOS) and COVID-19: an overlooked female patient population at potentially higher risk during the COVID-19 pandemic. BMC Med. 2020; 18 (1), 220.

[22] Mentzer R. GOP men are most likely to say they'll refuse COVID-19 vaccine. Available at: https://www. wpr.org/gop-men-are-most-likely-say-theyll-refusecovid-19-vaccine

[23] https: / / fivethirtyeight.com/features/why-is-theresuch-a-gender-gap-in-covid-19-vaccination-rates/. [accessed 03/07/2021].

[24] Ndugga N, Pham O, Hill L, Artiga S, Parker N. Latest data on COVID-19 vaccinations by race/ethnicity. Available at: https://www.kff.org/coronavirus-covid-19/issue-brief/latest-data-on-covid-19-vaccinations-race-ethnicity/. [accessed 03/07/2021].

[25] Jamison AM, Quinn SC, Freimuth VS. "You don't trust a government vaccine": Narratives of institutional trust and influenza vaccination among African American and white adults. Soc Sci Med. 2019; 221, 87-94.

[26] Freimuth VS, Quinn SC, Thomas SB, Cole G, Zook E, Duncan T. African Americans' views on research and the Tuskegee Syphilis Study. Soc Sci Med. 2001; 52, 797-808.

[27] Araojo R, Marks P. Vaccine Ready: Addressing COVID-19 Health Disparities among Racial and Ethinic Minority Communities. Available at: https: / / www.fda. gov / news-events / fda-voices / vaccine-ready-addressing-covid-19-health-disparities-among-racial-and-ethnic-minority-communities. [accessed 02/08/2021].

[28] Egede L, Walker R. Structural racism, social risk factors, and Covid-19 - a dangerous convergence for black Americans. N Engl J Med. 2020; 383, e77.

[29] Fernandez A. We need to get more Latinx people vaccinated. Here's how. Available at: https://www. aamc.org/news-insights / we-need-get-more-latinxpeople-vaccinated-heres-how. [accessed 18/06/2021].

[30] KFF. KFF COVID-19 vaccine monitor. Available at: https://www.kff.org/coronavirus-covid-19/dashboard/kff-covid-19-vaccine-monitor-dashboard/. [accessed 03/07/2021].

[31] Yan H. Covid vaccine: 10 reasons why young, healthy people need to get vaccinated - CNN. Available at: https://www.cnn.com/2021/05/05/health/young-people-covid-vaccine/index.html. [accessed 25/05/2021].
[32] Statista. Share of adult population enrolled in college or other higher education in the United States from 1970 to 2019, by age group. Available at: https:/ /www. statista.com/statistics/236093/higher-education-enrollment-rates-by-age-group-us/. [accessed 03/07/2021].

[33] Berman J. 'If a student chooses to come to an institution, they agree to abide by the rules': can colleges force students to get COVID-19 vaccines? Available at: https: / / www.marketwatch.com/story /if-a-studentchooses-to-come-to-an-institution-they-agree-to-abideby-the-rules-can-colleges-be-forced-to-mandate-covid19-vaccines-11617038227. [accessed 18/06/2021].

[34] Quinn SC, Kumar S, Freimuth VS, Kidwell K, Musa D. Public willingness to take a vaccine or drug under Emergency Use Authorization during the 2009 H1N1 pandemic. Biosecur Bioterror. 2009; 7, 275-90.

[35] Silverman H, Almasy S. CDC study finds coronavirus vaccines lead to milder disease in rare breakthrough infections. Available at: https://www.cnn. com/2021/06/07/health/us-coronavirus-monday / index.html. [accessed 09/06/2021].

[36] Havers FP, Whitaker M, Self JL, et al. COVID-NET Surveillance Team. Hospitalization of Adolescents Aged 12-17 Years with Laboratory-Confirmed COVID-19 COVID-NET, 14 States, March 1, 2020-April 24, 2021. MMWR Morb Mortal Wkly Rep. 2021; 70 (23), 851-7.

[37] Lardieri A. Pfizer applies for full FDA approval of coronavirus vaccine. Available at: https://www. usnews.com/news/health-news/articles/2021-05-07/ pfizer-applies-for-full-fda-approval-of-coronavirus-vaccine. [accessed 07/06/2021].

[38] Lovelace B. Jr. Moderna applies for full FDA approval of its Covid vaccine. Available at: https:/ /www. cnbc.com/2021/06/01/covid-vaccine-moderna-applies-for-full-fda-approval.html. [accessed 07/06/2021]. [39] Del Rio C. What does full FDA approval of COVID-19 vaccines actually mean? Available at: https:// thehill.com/opinion/healthcare/556567-what-doesfull-fda-approval-of-covid-19-vaccines-actually-mean. [18/06/2021].

[40] Meo SA, Bukhari IA, Akram J, Meo AS, Klonoff DC. COVID-19 vaccines: comparison of biological, pharmacological characteristics and adverse effects of Pfizer/BioNTech and Moderna Vaccines. Eur Rev Med Pharmacol Sci. 2021; 25 (3), 1663-9. 